\title{
Improvement the Laundry Folding System of Storing Laundry Process
}

\author{
Yuhki Kitazono $^{\mathrm{a}^{*}}$, Takumi Saruhashi ${ }^{\mathrm{a}}$, Seiichi Serikawa ${ }^{\mathrm{b}}$ \\ ${ }^{a}$ National Institute of Technology, Kitakyushu College,5-20-1 Shii, Kokuraminami-ku, Kitakyushu-city, Fukuoka \\ 802-0985, Japan \\ ${ }^{\mathrm{b}}$ Kyushu Institute of Technology, 1-1 Sensui-cho Tobata-ku Kitakyushu-city, Fukuoka 804-8550, Japan
}

*Corresponding Author:kitazono@kct.ac.jp

\begin{abstract}
In this paper, we discuss the laundry folding system that we have developed. This system has ability to take laundry off the hanger from laundry and fold laundry in automatically. After you take the laundry out of washing machine and put it on a hanger rack with the hanger, our system takes each hanger from the hanger rack, moreover detects whether the laundry is dry or not. After detecting that the laundry is already dry, this system removes the hanger from the laundry. Finally, this system folds the laundry. Folded laundry is stored on the place of the system. However, this system had a problem in this storing process. The problem is that this system didn't determine height of a pile of stored laundry. Therefore, if this system folds many laundries, the system couldn't store the laundry well. From this problem, we have improved this system. Improved system determines height of a pile of stored laundries. Based on the information of it height, the system could store the laundry well. By this improvement, the system became able to fold and store many laundries.
\end{abstract}

Keywords: folding the laundry, robot, folding device, Arduino, motor.

\section{Introduction}

Housework is one of tough jobs. Not only that, we must do it every day. We can't live comfortably without doing housework. In the case of a single life, you have to do that as well as your work. On the other hand, if you have a large family, amount of housework becomes enormous. There are a great number of household tasks such as cleaning the room, washing the dishes, and doing the washing ${ }^{(1,2)}$. Previously, housework has been done manually. However, as a result of appearance of Dishwasher, Vacuum cleaner, and Washing machine, we can conveniently save trouble. Nevertheless, the machine which folds the laundry automatically has not prevailed among the ordinary homes. Even today, folding the laundry is a manual labor.

In 2015, the laundry folding robot: "Laundroid" was developed by Seven Dreamers Laboratories, Inc., Panasonic Corporation, and Daiwa House Industry Co.,Ltd.. Though the robot has ability to fold many kind of clothes, it takes approximately 10 minutes to fold one T-shirt, and it doesn't consider whether the laundry has already dried or not. Due to that, user should check whether the laundry is dry when he takes the laundry in. Folding laundry in automatically has been studied by some researcher ${ }^{(3,4)}$. However, the laundry is dried in the laundry dryer, so hanging the laundry to dry process is ignored in previous studies. When we take in account the percentage of households owning a laundry dryer, hanging out the laundry to dry is yet main means to dry the laundry ${ }^{(5,6)}$. Addition to this, some clothes can't be dried in the laundry dryer because they shrink in the heat of the dryer. Also there are some attempts to keep a proper humidity of the room by hanging out the laundry to dry in the room to prevent the room from drying ${ }^{(7,8)}$. If we hanged the laundry out, we have to detect whether the laundry is dried or not when we take the laundry in. After that, we have to remove the hanger from laundry to fold laundry. However, these processes of folding laundry are not much studied.

From such background, we had developed the system to fold laundry that state hanged out. The developed system could detect whether the laundry is dry or not. After detecting that the laundry is already dry, the system removes the hanger from the laundry and folds the laundry. Folded laundry is stored on the place of the system. However, the system hadn't determined height of a pile of stored laundry in this storing process. Therefore, the system 


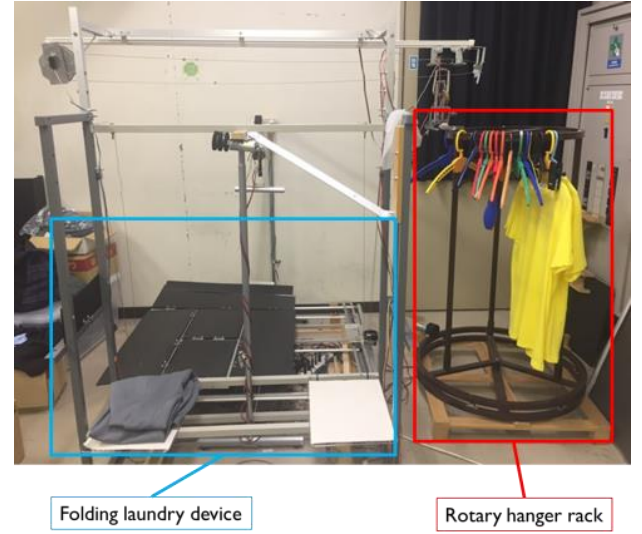

Fig. 1. Overall view of the system..

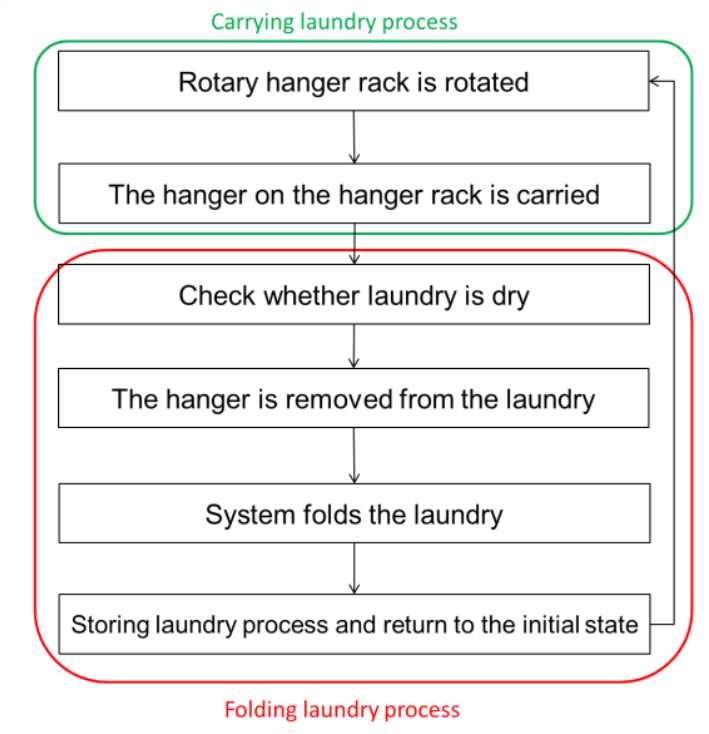

Fig. 2. The outline of the system.

couldn't store many laundries.

Therefore, we have improved the folding laundry system. We had reviewed the laundry storing process of the system. Moreover, we have attached the sensor and changed programs that control motor as improvement.

\section{Construction of the System}

\subsection{System overall view and the operating procedure}

The overall view of the system is shown in Fig. 1. The rotary hanger rack part is shown in right side in the Fig. 1. The user hangs laundry on the rotary hanger rack. We use the clothes pin (NSA-37 by NAITO-KOGYOSHO, INC.) and hanger (DFQ011 by DAISAKU-SHOJI, INC) to hang laundry. We pinch the laundry to clothespins of the hanger. If the laundry has a sleeve such as a shirt, we hang the laundry to the hanger in normally. So, the sleeve is put through by the wings of the hanger. Folding laundry device part is shown the left side in the Fig. 1. This device is the main part of the system. It detects dryness of laundry, removes hanger from laundry, and folds laundry. The behavior outline of the system is shown in Fig. 2. The behaver of the system can roughly divide into two parts: carrying laundry process and folding laundry process. In carrying hanger process, the system makes the laundry moves from rotary hanger rack. Thereafter, the system removes hanger from laundry. Finally in this process, the laundry placed on the laundry folding device. After that, the system runs the folding process and controls laundry folding device. In the folding laundry process, the system folds the laundry using laundry folding device. After the system folding laundry, the system stores the laundry on the place of the system. By using motors and operating the respective device, these operations are realized. Also state of each device is detected by sensors and motors, and they are controlled by Arduino. AC100V is used for the power supply of the system. It is converted to DC5V and DC12V by the power strip. The converted voltage is supplied to each device.

\subsection{Problem in storing the laundry process}

The laundry is folded by the laundry folding device as shown in Fig. 3. Folded laundry is stored on the place of the system. The system stores folded laundry using folding laundry device as shown in Fig. 4. Folded laundries were piled up by the system. However, the system didn't determine height of top of a piled of laundry. Therefore, if the system folds many laundries, the system couldn't store laundries well.

\subsection{Improvement storing the laundry process}

We attached distance sensor to determine height of a pile of stored laundries as shown in Fig. 5. The information of height is processed by Arduino. Based on the information of a pile of laundry height, Arduino controls the motor that adjust the height of the folding laundry device. By this improvement, the system became able to control the height of folding laundry device depending on the height of a pile of laundry. The system controls device height to the same height as the pile of laundry. 


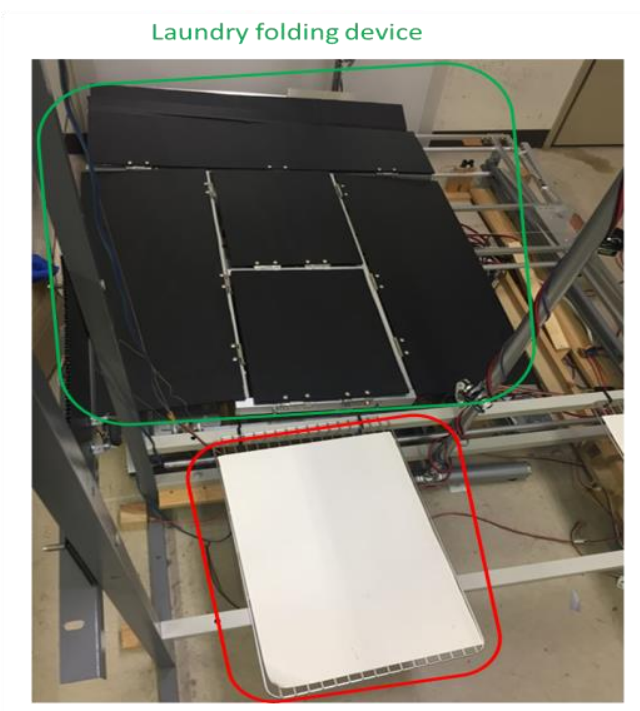

Storing place

Fig. 3. Laundry folding device.

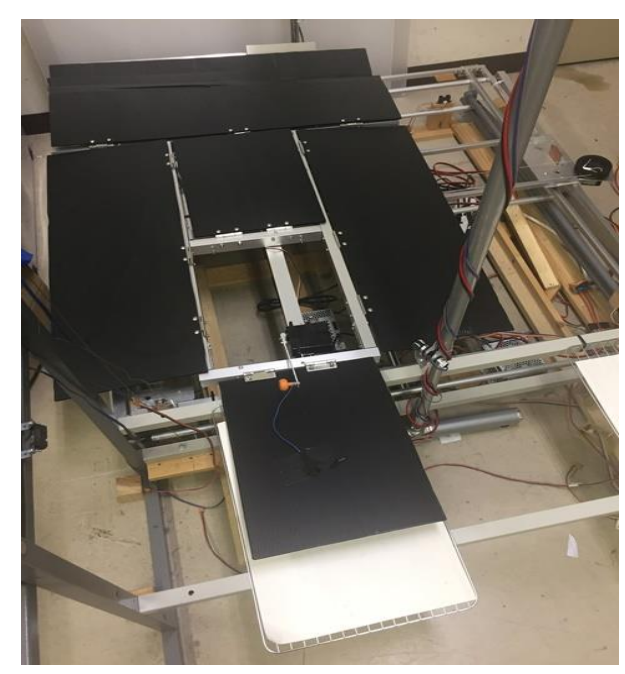

Fig. 4. Behavior of the laundry folding device stores laundry.

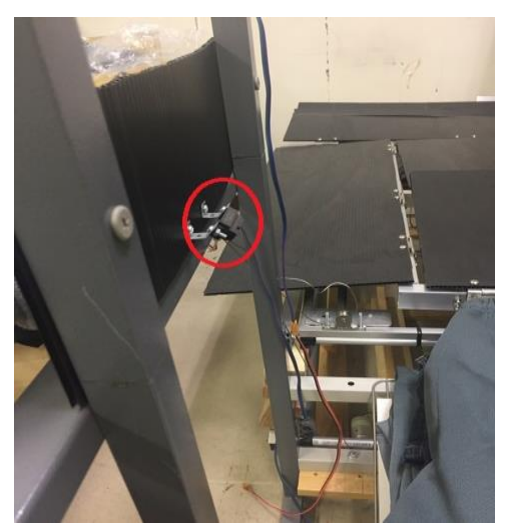

Fig. 5. Attached distance sensor.

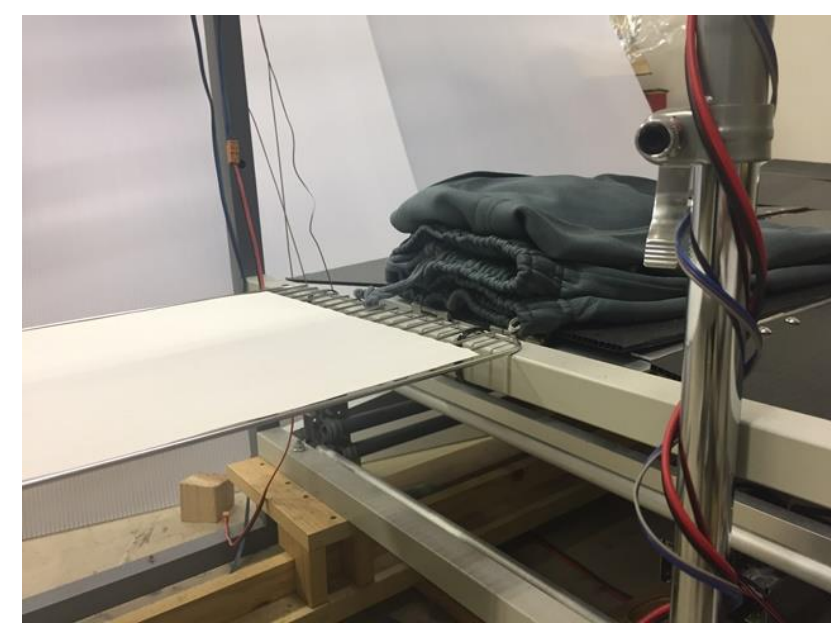

(a) Height adjustment.

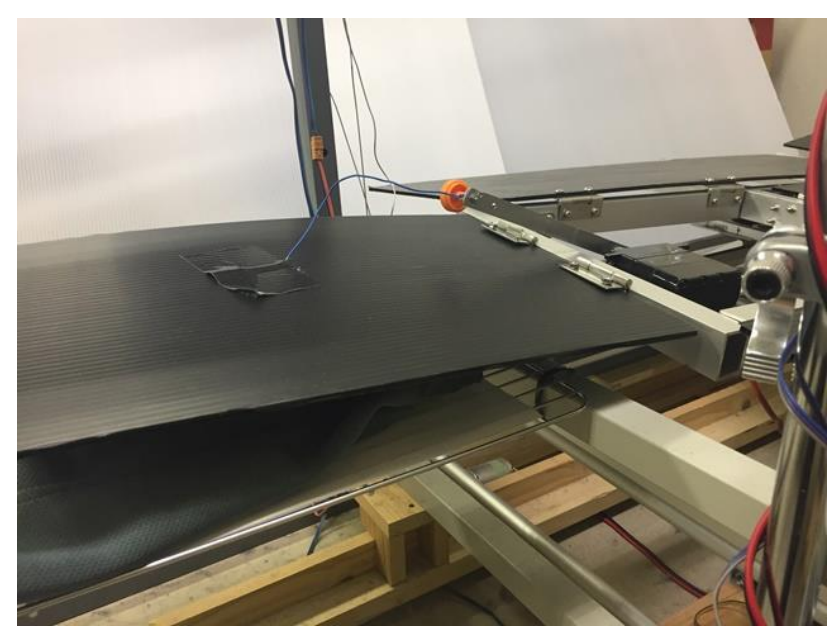

(b) Storing the laundry.

Fig. 6. Cases of no laundries are already stored.

\section{Experiment}

We checked the performance of the folding laundry device that we have improved. We have made the system fold laundries and store them. The result of experiment, the system could determine height of the stored laundry. Moreover, the system could adjust the height of folding device depending on the height of stored laundry as shown in Figs. 6, 7. Comparing Fig. 6 with Fig. 7, the position of laundry folding device is changed. The height of the device is controlled depending on the height of already stored laundries. Finally, the system could store the folded laundry. The system could store about 20 laundries in this experiment. It amount is enough when considering the limit amount of we can hang the laundry to rotary hanger rack. 


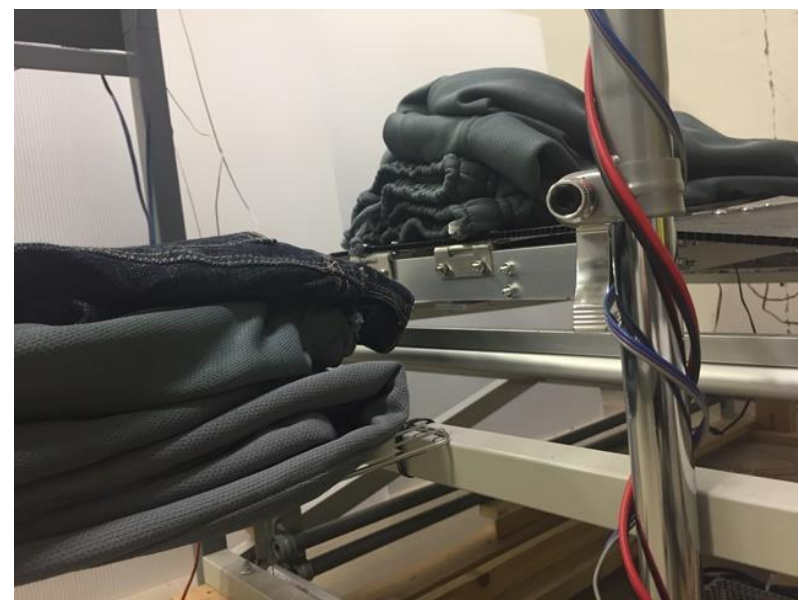

(a) Height adjustment.

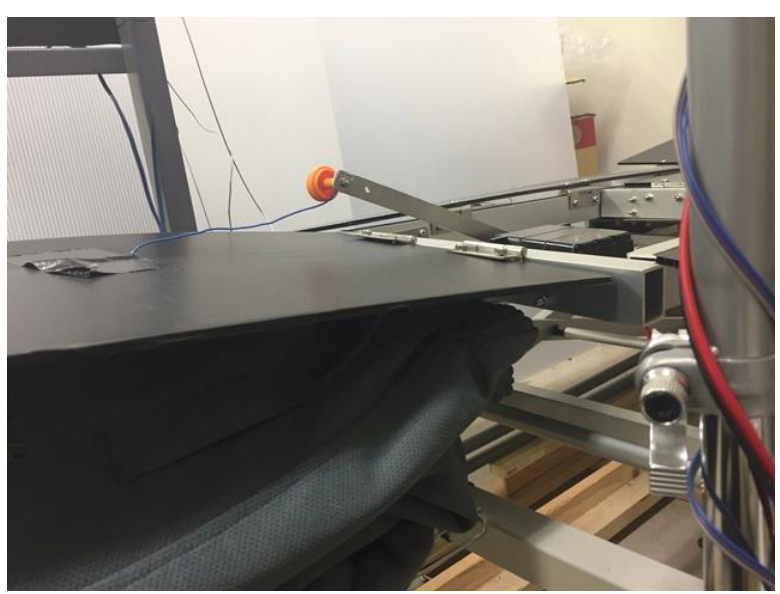

(b) Storing the laundry.

Fig. 7. Cases of laundries are already stored.

\section{Conclusions}

We have improved the laundry folding system to store the many laundries. We have attached the distance sensor to the place that the folded laundry is stored. Improved system could determine height of a pile of stored laundries. In the experiment, the system could height adjust and store the laundry well. By this improvement, the system became able to fold and store many laundries.

However, we still have problems. Future issue of our study is folding the small size laundry, for example, socks, handkerchief, and so on.

\section{References}

(1) Kabata Shinichiro : "Technological Progress of Washing Machine", Journal of Japan Society of Mechanical Engineers, Vol. 115, No. 1120, pp. 146-147,
2012

(2) Nishiwaki S. : "Tecqnology Trend of Washing Machine", Journal of Japan Society of Mechanical Engineers, Vol. 115, No. 1120, pp. 146-147, 2012

(3) Hamajima Kyoko, Kakikura Masayoshi : "Planning Strategy for Task of Utangling Laundry - Isolation of Clothes from Washed Mass -", JRM, Vol. 10, No. 3, pp. 244-251, 1998

(4) Kaneko Manabu, Kakikura Masayoshi : "Study on Handling Clothes (Task Planning of Deformation for Unfolding Laundry)” JRM, Vol. 15, No. 4, pp. 406-415, 2003

(5) Fukuda Eiko : "Survey of Home Washing", The journal of Wayo Women's University, Vol. 43, pp. 85-98, 2003

(6) Koitabashi Emiko, Okita Fumiko: "The Actual Conditions of Washing Work/Working Space and Problems to Be Solved : From an Investigation Carried Out in 2000 and in 2008", Memoirs of the Japan Women's University. Faculty of Home Economics, Vol. 57, pp. 95-106, 2010

(7) Kazue Shoushi, Sato Manami : “41159 Study on control methods of relative humidity in a room to use desorption from finishing material and clothes : Part 1 An evaluation of hygrothermal distribution by actual measurement", Summaries of technical papers of Annual Meeting Architectural Institute of Japan. D-2, Environmental engineering II, Heat, moisture, thermal comfort, natural energy, air flow, ventilation, smoke exhaustion, computational fluid dynamics, indoor air quality heating, cooling and air-conditioning heat and cold sources, piping systems application of building services 2008, pp. 317-318, 2008

(8) Kazue Shoushi, Sato Manami: “41160 Study on control methods of relative humidity in a room to use desorption from finishing material and clothes : Part 2 An numerical analysis of hygrothermal distribution", Summaries of technical papers of Annual Meeting Architectural Institute of Japan. D-2, Environmental engineering II, Heat, moisture, thermal comfort, natural energy, air flow, ventilation, smoke exhaustion, computational fluid dynamics, indoor air quality heating, cooling and air-conditioning heat and cold sources, piping systems application of building services 2008, pp. 319-320, 2008 